\title{
Secondary alopecia neoplastica - the first metastasis of the breast cancer
}

\section{Introduction}

Secondary alopecia neoplastica( $\mathrm{AN}$ ) represents metastases of internal malignancies in scalp skin. Clinically, it is most commonly seen as one or more areas of cicatricial alopecia. Although at first it may resemble alopecia areata, later, induration, telangiectasia, nodular lesions may be seen. ${ }^{1}$ AN may correspond to the initial clinical presentation of hidden internal malignancies but may also indicate a neoplasia relapse in previously stabilized oncological patients .Brownstein found that the scalp metastases account for $4 \%$ of allcutaneous metastases. ${ }^{2}$

Herein, we show AN as the first relapse of the breast cancer 6 years after surgery.

\section{Case report}

A 55-year - old woman was referred to our outpatients clinic because of alopecic plaques over the scalp that appeared 6 months earlier. She has a history of right breast cancer surgery (carcinoma ductale invasivum HG II, NG II, Pt 12mm ) 6 years ago, followed by chemotherapy and radiation therapy.

On examination, in parietal region two alopecic plaques, measuring $4 \times 5 \mathrm{~cm}$ i $2 \times 2 \mathrm{~cm}$, slightly sclerotic, erythematous, shiny, with rare crusts and telangiectasia were seen (Figure 1a). Routine laboratory analyses and tumor markers( CA 128, CA 15-3, CEA ) were within normal limits, chest $\mathrm{x}$-ray and ultrasound of the abdomen showed no pathological lesions.
Volume 5 Issue 5 - 202।

Jelica Vukicevic

Medical faculty of Belgrade, University of Belgrade, Serbia

Correspondence: Jelica Vukicevic, Clinic of

Dermatovenereology, Clinical center of Serbia, Belgrade, Pasterova 2, Serbia, Tel +38I I I 3662457

Email jvukicevicsretenovic@gmail.com

Received: September 30, 202 I | Published: October 28, 2021

Histopathology of the by opted skin lesion revealed atypical cells that form infiltrative cords, small lobules and ducts throughout the dermis. These cells exhibited marked pleomorphism with high nuclear/ cytoplasmic ratio (Figure 2). Metastatic breast carcinoma was confirmed.

MSCT of the endocranium, thorax and abdomen was done and no metastatic changes were found. WB skeleton scintigraphy revealed no pathological changes.

Treatment was started with tamoxifen $2 \times 20 \mathrm{mg}$ /day plus palliative radiotherapy for the first 4 months, and then with tamoxifen only. One year later, there was a significant clinical regression of the metastatic scalp lesion (Figure 1b).

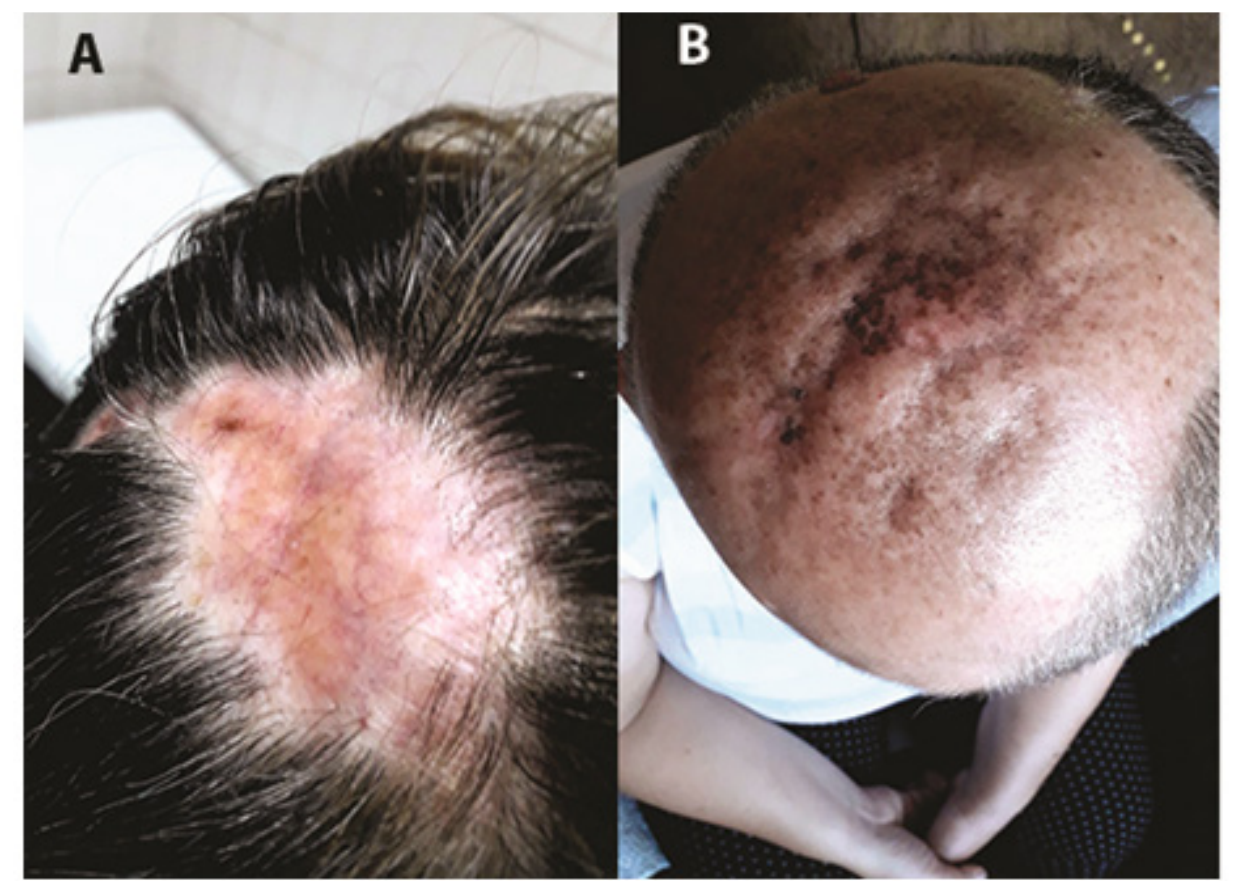

Figure I Alopecianeoplastica - before (A) and after treatment. 


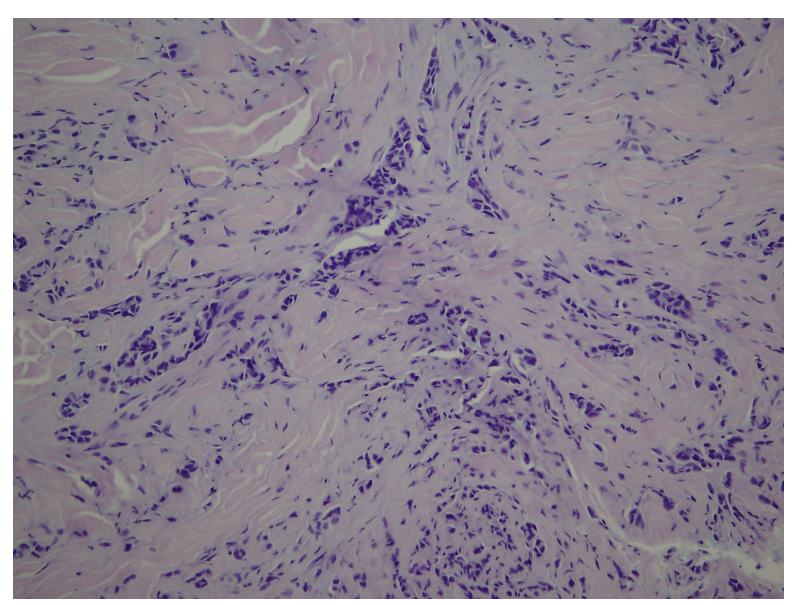

Figure 2 Histopathology: a typical cells that form infiltrative cords, small lobules and ducts throughout the dermis (HE x200).

\section{Discussion}

AN is a term established by Cohen in 1961 and represents a rare form of cutaneous metastasis where an underlying cancer spreads to the scalp. ${ }^{1}$ The lesions of ANare most commonly localized in the parietal and frontal regions. It seems that regulatory genes, growth factors and cell-cell interactions may influence the biology of these two scalp regions because of their dermal origin. ${ }^{3}$ According to some authors, the affinity of cancer cells to the scalp skin seems to be an inherent characteristic of the individual neoplasm. ${ }^{4}$ There is also an opinion accepted by most authors that scalp affords a high degree of vascularity, immobility, and warmth, which enhances affinity of cancers cells for this region.

Spreading of cancer cells occurs through adjacent lymphatics, lymphatic or hematogenous emboli, by direct invasion of the skin from underlying tumor or accidentally. ${ }^{6}$

The mechanism of hair loss in AN has not been clarified to date. Scarring may be the result of desmoplasia which is related to the presence of basic fibroblast growth factor, interleukin 4 and 6 , inflammatory mediators, that are released by neoplastic cells.7 It is known that breast carcinoma, desmoplastic melanoma, as well as gastric carcinoma, have a prominent collagenous stroma. Factors independent of fibrosis, such as tumor invasion of the hair sheaths, may play a role in the development of alopecia, because hair may be regrown in alopecic areas after effective cancer treatment.

Recently, AN is divided into primary, when the cancer originates in the cutaneous scalp and secondary, when the malignancy originates from a visceral organ, and has metastasized to the scalp skin. ${ }^{8}$

According to the results of Palino and coworkers, who conducted a systematic review concerning histological confirmed cases of secondary AN, women were represented with $53,7 \%$ of cases. Median age of AN at onset was 59.Regarding clinical appearance, AN was identified as a nodule, plaque and patch in 58\%, 9,2\% and 13,4\% respectively. Gastrointestinal tract is the most frequently reported site of primary malignancy $(24.4 \%)$ followed by breast cancer accounted for $17,9 \% .^{3}$

In women, the most common source of AN is the breast cancer, and metastases usually occured late in the disease. Surely, the scalp metastases may be the initial sign of malignant disease when it is expected that primary cancer is localized in the lung $(60 \%)$, the kidney $(53 \%)$ or the ovary $(40 \%){ }^{9}$
AN originating from breast cancer commonly clinically presents as one to two patches or plaques with an average size of about $3 \mathrm{~cm}$. Plaques are indurated, erythematous, telangiectatic which is significant for eliminating alopecia areata. Certainly, the clinical presentation also depends on the developmental stage of metastatic lesion. Initially, it may look like alopecia areata, and in the later phase, tumor masses were written. ${ }^{1}$ However, AN may resemble other forms of alopecia. Thus, Gül U. et all, described AN due to metastatic colon adenocarcinoma represented as subtotal alopecia.10 Lim HLJ. et coworkers presented a case of multifocal AN in a Chinese woman one year after right breast mastectomy and adjuvant chemotherapy because of Stage 3 invasive ductal carcinoma. ${ }^{11}$

It is important to emphasize that adenocarcinoma is the most common cancer histological subtype presenting metastases toward skin. ${ }^{12}$

Different therapeutic options for AN are being implemented: hemiotherapy, radiation therapy, surgical excision.

Cutaneous metastases are known to identify with advanced visceral malignancy and to be a poor prognostic sign. Recent research studies suggest that the survival period in patients with cutaneous metastases depends on the localization of visceral malignancy. Thus, the survival period is longer for breast cancer patients than for patients with lung cancer or other kinds of neoplasia. Interestingly, despite the fact that AN is very rare, according to the recent study results, it wasthe first metastatic event in $65,3 \%$ of patients with visceral malignancies. ${ }^{3}$

Herein, we presented a patient with AN as the first metastasis 6 years after breast cancer operation with good outcome to tamoxifen and radiotherapy. One year after the diagnosis of $\mathrm{AN}$, no new metastases were found and the patient was in good general condition.

Diagnosis of AN may be challenging, especially when its onset precedes the diagnosis of the primary malignancy. Biopsy of scarring alopecia, especially in patient with history of internal malignancy is obligatory.

\section{Conflicts of interest}

The authors declare no conflict of interest.

\section{Acknowledgments}

None.

\section{Funding}

None.

\section{References}

1. Cohen I, Levy E, Schreiber H. Alopecia neoplastica due to breast carcinoma. Arch Dermatol. 1961;84:490-492.

2. Brownstein MH, Helwig EB.Patterns of cutaneous metastasis. Arc Dermatol. 1972;105:862-868.

3. Paolino G, Pampena R, Grassi S, et al. Alopecia neoplastica as a sign of visceral malignancies: a systematic review. JEADV. 2019;33(6):1020 1028.

4. Mohs FE, Lathrop TG. Modes of spread of cancer of the skin. Arch Dermatol Syph.1952;66:427-439.

5. Gates O. Cutaneous metastases of malignant disease. Am J Cancer. 1937;30:718-730.

6. Mehregan AH. Metastatic carcinoma to the skin. Dermatologica. $1961 ; 123: 311-325$ 
7. Johnson WC. Metastatic carcinoma of the skin. In: Elder D, Elenitas R, Jaworski C, Johnson BJr, editors. Lever Histopathologz of the skin, 10th edn. Phiadelphia, PA: Lippincott. 2009:1155-1156.

8. Cohen PR. Primary alopecia neoplastica versus secondary alopecia neoplastica: a new classification for neoplasm- associated scalp hair loss. J Cutan Pathol. 2009;36:917-918.
9. Gül U, Kili A, Akbas A, et al. Alopecia neoplastica due to metastatic colon adenocarcinoma. Acta Dermatol Venereol. 2006.

10. Lim HLJ, Siow SW, Tan SL.A case of multifocal scaring alopecia. Int J Dermatol. 2017; 56:993-995.

11. Rondina A, Kohen LL, Scherschun L, et al. A woman with focal alopecia. J Am Acad Dermatol. 2010;63(3):545-547. 